\title{
Brain-derived neurotrophic factor reduces inflammation and hippocampal apoptosis in experimental Streptococcus pneumoniae meningitis
}

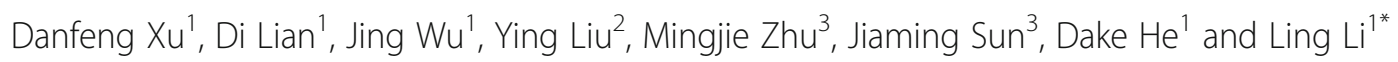

\begin{abstract}
Background: Streptococcus pneumoniae meningitis is a serious inflammatory disease of the central nervous system (CNS) and is associated with high morbidity and mortality rates. The inflammatory processes initiated by recognition of bacterial components contribute to apoptosis in the hippocampal dentate gyrus. Brain-derived neurotrophic factor (BDNF) has long been recommended for the treatment of CNS diseases due to its powerful neuro-survival properties, as well as its recently reported anti-inflammatory and anti-apoptotic effects in vitro and in vivo.

Methods: In this study, we investigated the effects of BDNF-related signaling on the inflammatory response and hippocampal apoptosis in experimental models of pneumococcal meningitis. Pretreatment with exogenous BDNF or the tropomyosin-receptor kinase B (TrkB) inhibitor k252a was performed to assess the activation or inhibition of the BDNF/TrkB-signaling axis prior to intracisternal infection with live S. pneumoniae. At $24 \mathrm{~h}$ post-infection, rats were assessed for clinical severity and sacrificed to harvest the brains. Paraffin-embedded brain sections underwent hematoxylin and eosin staining to evaluate pathological severity, and cytokine and chemokine levels in the hippocampus and cortex were evaluated by enzyme-linked immunosorbent assay. Additionally, apoptotic neurons were detected in the hippocampal dentate gyrus by terminal deoxynucleotidyl transferase dUTP-nick-end labeling, key molecules associated with the related signaling pathway were analyzed by real-time polymerase chain reaction and western blot, and the DNA-binding activity of nuclear factor kappa B (NF-KB) was measured by electrophoretic mobility shift assay.
\end{abstract}

Results: Rats administered BDNF exhibited reduced clinical impairment, pathological severity, and hippocampal apoptosis. Furthermore, BDNF pretreatment suppressed the expression of inflammatory factors, including tumor necrosis factor $\alpha$, interleukin (IL)-1 $\beta$, and IL-6, and increased the expression of the anti-inflammatory factor IL-10. Moreover, BDNF pretreatment increased TrkB expression, activated downstream phosphatidylinositol 3-kinase (PI3K)/protein kinase B (AKT) signaling, and inhibited the myeloid differentiation primary response gene 88 (MyD88)/NF-kB-signaling pathway.

Conclusions: These data suggested that BDNF administration exerted anti-inflammatory and anti-apoptotic effects on an experimental pneumococcal meningitis model via modulation of MyD88/NF-KB- and PI3K/AKT-signaling pathways. Our results indicated that treatment with exogenous BDNF might constitute a potential therapeutic strategy for the treatment of bacterial meningitis.

Keywords: Streptococcus pneumoniae meningitis, Brain-derived neurotrophic factor, Neuroinflammation, Hippocampal apoptosis

\footnotetext{
* Correspondence: linglidoctor@163.com

'Department of Pediatric Neurology, Xinhua Hospital affiliated to Shanghai

Jiaotong University School of Medicine, Kongjiang Rd 1665, Shanghai

200092, People's Republic of China

Full list of author information is available at the end of the article
} 


\section{Background}

Bacterial meningitis is a severe infection of the central nervous systems (CNS), with an annual occurrence of 0.9 per 100,000 people in developing countries $[1,2]$. The most common causative agent is Streptococcus pneumoniae, with a case fatality rate of $\sim 30 \%$ in developed countries and nearly 50\% in less-developed countries [3-5]. Moreover, $\sim 50 \%$ of survivors suffer from persistent neurological sequelae throughout their life, including learning and memory deficits, seizures, and hearing impairment $[6,7]$. The inflammatory response plays a vital role in disease pathogenesis, with bacterial compounds recognized by brain-resident immune cells capable of recruiting myeloid differentiation factor 88 (MyD88) and inducing nuclear translocation of nuclear factor kappa $\mathrm{B}$ (NF-kB), followed by the production of inflammatory mediators $[8,9]$. This enhanced inflammatory response triggered to eliminate bacterial components exerts both defensive and neurotoxic effects [10]. Although therapies necessary to reduce inflammation are required, neurological sequelae associated with the disease correlate with hippocampal apoptosis caused by both the bacterial toxins and the intensive immune response [10]. A single treatment capable of reducing both inflammation and hippocampal apoptosis could potentially improve outcomes in children with $S$. pneumoniae meningitis.

Brain-derived neurotrophic factor (BDNF) is a member of the neurotrophic family, which plays an important role in the development, differentiation, and survival of neurons in the CNS $[11,12]$. BDNF exerts neuroprotective effects in multiple CNS diseases following its high-affinity binding to tropomyosin-receptor kinase B $(\operatorname{TrkB})[13,14]$. In recent years, significant effort has been expended to identify the neuroprotective effects of BDNF on S. pneumoniae meningitis in both animal experiments and clinical studies. Our previous study reported the levels of BDNF and its receptor TrkB increased following acute $S$. pneumoniae meningitis but subsequently declined over time, especially following administration of antibiotics [15]. Similarly, increased BDNF levels were also observed in the serum and cerebrospinal fluid (CSF) of pediatric patients with CNS infections on the day of admission [16]. Increased BDNF synthesis during the acute phase of meningitis could stimulate proliferation of dentate granule cells and promote neurogenesis after bacterial meningitis [17]; however, this self-reparative capacity is insufficient, given that most newly generated cells are unable to differentiate into immature neurons and neurons in experimental $S$. pneumoniae meningitis [9], which worsens as BDNF decreases over time. Additionally, Barichello et al. [18] reported that decreases in BDNF levels during the long-term phase of meningitis were correlated with behavioral deficits in adult animals submitted to meningitis during the neonatal period. Interestingly, our previous study reported that administration of exogenous
BDNF increased rates of neuron survival [18], and it was recently reported that exogenous BDNF increases neurogenesis of neuron stem cells in the hippocampus after $S$. pneumoniae meningitis [9]. In addition to its neuroprotective effects, BDNF participates in anti-inflammatory and anti-apoptotic processes according to a study of experimental allergic encephalomyelitis [13]. Furthermore, BDNF can attenuate ischemic-hypoxic injury by modulating local inflammation in rats suffering from ischemic stroke [19]. Taken together, these findings indicate BDNF involvement in regulating inflammatory processes; however, the mechanisms associated with BDNF signaling related to these responses remain unknown. BDNF-related neuroprotective effects are elicited by activation of extracellular signalrelated kinase (ERK)- and phosphatidylinositol 3-kinase $(\mathrm{PI} 3 \mathrm{~K}) /$ protein kinase $\mathrm{B}(\mathrm{AKT})$-signaling pathways, and recent evidence suggests that PI3K participates in negative regulation of inflammatory pathways [13, 20, 21]. However, the contribution of this signaling pathway to BDNF-associated prevention of brain injury related to S. pneumoniae meningitis remains unclear.

Here, we explored whether BDNF/TrkB interaction modulates localized inflammation in the infected brain by exerting neuroprotective effects through reductions in hippocampal apoptosis associated with S. pneumoniae meningitis. We further investigated whether these effects are mediated by the MyD88/NF-kB- and PI3K/AKT-signaling pathways.

\section{Methods}

\section{Animals and cannula implantation}

Three-week-old female Sprague-Dawley rats (50-55 g) were obtained from the Shanghai Laboratory Animal Management Center (Shanghai, China). Cannula implantation was undertaken based on previously described procedures [9]. Briefly, one stainless steel cannula was implanted into the right lateral cerebral ventricle after the rat was anesthetized with $10 \%$ chloral hydrate $(0.15-$ $0.3 \mathrm{~mL}$ per $100 \mathrm{~g}$, administered intraperitoneally). The location of the cannula implantation was $3.8 \mathrm{~mm}$ rostral to the lambdoid suture of the skull, $2 \mathrm{~mm}$ lateral to the right side from the midline of the skull, and $2.5 \mathrm{~mm}$ from the skull surface. Following surgery, all rats were returned to their cages to allow a 3-day recovery. Rats were housed under a 12-h light/dark cycle, with food and water available ad libitum. Animal experiments were approved by the Animal Ethical and Welfare Committee of Xinhua Hospital affiliated to Shanghai Jiaotong University School of Medicine. All efforts were made to minimize the number of animals used and their suffering.

\section{Infecting organisms}

The standard strain of serotype III S. pneumoniae was obtained from American Type Culture Collection (Manassas, 
VA, USA). The bacterial strain was cultured overnight on a blood agar plate, followed by inoculation into Vital Aer Broth (R\&D Systems, Minneapolis, MN, USA) and incubation at $37{ }^{\circ} \mathrm{C}$ under $5 \% \mathrm{CO}_{2}$ for $18 \mathrm{~h}$ to reach the logarithmic phase as reported previously [22]. Bacteria were centrifuged for $15 \mathrm{~min}$ at $5000 \mathrm{~g}$ and re-suspended in sterile saline solution to an approximate concentration of $1 \times 10^{4} \mathrm{CFU} / \mathrm{mL}$ by using a nephelometer (bioMerieux, Marcy-l'Étoile, France).

\section{Animal model of S. pneumoniae meningitis}

Bacterial inoculations were performed under anesthesia. The rats underwent an intracisternal puncture to remove $20 \mu \mathrm{L}$ of CSF, followed by intracisternal injection of a $20-\mu \mathrm{L}$ volume containing either $1 \times 10^{4} \mathrm{CFU} / \mathrm{mL} S$. pneumoniae or pyrogen-free saline. At $24 \mathrm{~h}$ postinoculation, all infected rats developed meningitis according to clinical evaluation, which was confirmed by quantitative analysis of culturing $5 \mu \mathrm{L}$ of CSF obtained through a puncture of the cisterna magna. The rats were weighed, and the severity of the disease was assessed clinically using the following scores: 1 = coma; 2 = does not turn upright when positioned on the back; 3 = turns upright within $30 \mathrm{~s} ; 4=$ minimal ambulatory activity, turns upright within $<5 \mathrm{~s}$; and $5=$ normal [23].

\section{Experimental design}

Rats $(n=66)$ were included in this study and randomized into sham and pneumococcal meningitis (PM) groups. The rats in the sham group $(n=10)$ were inoculated intracisternally with normal saline, whereas the rats in the PM groups $(n=56)$ were inoculated intracisternally with $S$. pneumoniae and further randomized into four groups ( $n=14$ each) according to the administration of different pretreatments. Prior to induction of meningitis as described above, the rats received an injection once daily for four consecutive days of $7.5 \mu \mathrm{L}$ recombinant BDNF $(6 \mu \mathrm{g} /$ day and diluted in phosphate-buffered saline (PBS; pH 7.4); PeproTech, Rocky Hill, NJ, USA) [9] or the TrkB inhibitor $\mathrm{k} 252 \mathrm{a}(1 \mu \mathrm{g} /$ day and diluted in $10 \%$ dimethyl sulfoxide (DMSO) in PBS; Sigma-Aldrich, St. Louis, MO, USA) [24] through a cannula previously implanted in the cerebral ventricle. The equivalent volume of PBS or $10 \%$ DMSO was injected into the vehicle control groups. All surviving animals were sacrificed by decapitation at $24 \mathrm{~h}$ post-inoculation to harvest the brains. For histological analysis, the animals were perfused through the heart with $50 \mathrm{~mL}$ of normal saline, followed by $200 \mathrm{~mL}$ of $4 \%$ paraformaldehyde (PFA) in PBS, and the brains were removed and post-fixed in $4 \%$ PFA overnight at $4{ }^{\circ} \mathrm{C}$. For biochemical analysis, the brains were dissected immediately, and the cortex and hippocampus were isolated and stored at $-80{ }^{\circ} \mathrm{C}$.
Tissue pathology and terminal deoxynucleotidyl transferase dUTP-nick-end labeling (TUNEL) staining Brain tissues were processed, embedded in paraffin, and cut into coronal sections of 4- $\mu$ m thickness. Hematoxylin and eosin (H\&E; Beyotime, Beijing, China) staining was performed according to a standard protocol, and apoptosis in the hippocampus was examined by TUNEL immunofluorescence staining using an in situ cell death detection kit (Roche, Basel, Switzerland) according to manufacturer instructions.

\section{Enzyme-linked immunosorbent assay (ELISA)}

Brain tissues (hippocampus and cerebral cortex) were homogenized in PBS buffer containing a proteinase inhibitor (100 mg of tissue per milliliter) and centrifuged at $12,000 \mathrm{~g}$ for $15 \mathrm{~min}$. The concentrations of tumor necrosis factor- $\alpha$ (TNF- $\alpha$ ), interleukin (IL)-1 $\beta$, IL-6, and IL-10 in the supernatant were examined using commercially available ELISA kits (eBioscience, San Diego, CA, USA) according to manufacturer protocols. Cytokine and chemokine concentrations in homogenates were normalized to total brain weight and reported as pg/ $100 \mathrm{mg}$ of tissue.

\section{Electrophoretic mobility shift assay (EMSA)}

Nuclear extract preparation and EMSA were performed using an EMSA kit (Thermo Fisher Scientific, Waltham, MA, USA) to determine NF- $\mathrm{BB}$ DNA-binding activity. Briefly, an NF- $\mathrm{B}$ oligonucleotide probe (forward: 5 ' $-\mathrm{AGT}$ TGA GGG GAC TTT CCC AGG C-3'; reverse: 5'-G CCT GGG AAA GTC CCC TCA ACT-3') was endlabeled with p65 biotin, and $20 \mu \mathrm{g}$ of nuclear extract was incubated with binding buffer and nonspecific oligonucleotides at room temperature for $15 \mathrm{~min}$, followed by incubation with a p65 biotin-labeled oligonucleotide complex for an additional $15 \mathrm{~min}$. Samples were subsequently separated by electrophoresis in $5.5 \%$ polyacrylamide gels in $0.25 \times$ Tris-borate-EDTA buffer. Following separation, the bands were detected by chemiluminescence.

\section{RNA extraction and real-time polymerase chain reaction (PCR)}

Total RNA from brain tissues (hippocampus and cerebral cortex) was extracted using Trizol reagent (TaKaRa, Shiga, Japan) and isolated using chloroform according to manufacturer instructions. RNA was converted to complementary DNA (cDNA) using the PrimeScript One Step RT-PCR kit (TaKaRa). Real-time PCR was performed on an ABI7500 system (Applied Biosystems, Carlsbad, CA, USA) using the SYBR Premix Dimmer Eraser kit (TaKaRa) to amplify the cDNA template. The primer sequences were as follows: $\beta$-actin forward 5'-GACAGGATGCAGAAGGA GATTACT-3' and reverse 5'-TGATCCACATCTGCTG GAAGGT-3'; MyD88 forward 5'-GATCCCACTCGCAG 
TTTGTT-3' and reverse 5'-GATGCGGTCCTTCAGTT CAT-3'; and TrkB forward 5'-GCTTCTGGAGGGCT TCTCTT-3' and reverse 5'-TGTTCTCTGGGTCAATGC TG-3'. The primers were synthesized by Shanghai Sangon Biological Engineering Technology Company (Shanghai, China). MyD88 and TrkB gene expression in each sample was normalized to $\beta$-actin expression, and the relative expression of messenger RNA (mRNA) was calculated using the $2^{-\Delta \Delta \mathrm{Ct}}$ method.

\section{Western blot}

Brain tissues (hippocampus and cortex) were lysed with radioimmunoprecipitation assay buffer containing $1 \mathrm{mM}$ phenylmethylsulfonyl fluoride. The lysates were centrifuged at $12,000 \times g$ for $15 \mathrm{~min}$ at $4{ }^{\circ} \mathrm{C}$, and the protein concentration of each sample was determined using a BCA protein assay kit (Beyotime) according to manufacturer instructions. Protein samples $(50 \mu \mathrm{g})$ were boiled for $10 \mathrm{~min}$, electrophoresed on $10 \%$ sodium dodecyl sulfate polyacrylamide gels, and transferred to polyvinylidene fluoride membranes. The membranes were blocked with 5\% skimmed milk in Tris-buffered saline and Tween-20 (TBST) for $2 \mathrm{~h}$ at room temperature. The membranes were then incubated at $4{ }^{\circ} \mathrm{C}$ overnight with the following primary antibodies: anti-phospho-PI3K, anti-PI3K, anti-phospho-AKT, anti-AKT, and antiMyD88 (1:1000; Cell Signaling Technology, Danvers, MA, USA), and anti-TrkB (1:1000; Abcam, Cambridge, UK). The membranes were washed with TBST three times for $10 \mathrm{~min}$ each and incubated with the appropriate secondary antibody for $1 \mathrm{~h}$. Proteins were visualized by chemiluminescence (Millipore, Billerica, MA, USA) and quantitated using ImageJ software (National Institutes of Health, Bethesda, MD, USA).

\section{Statistical analysis}

Statistical analysis was performed using one-way analysis of variance for parametric data, followed by Tukey's post hoc test; otherwise, the Mann-Whitney test was used. Survival rates were compared using the log-rank test. If not stated otherwise, values were expressed as the mean \pm standard deviation. Differences were considered significant at $p<0.05$. All graphs were constructed using GraphPad Prism 5.0 software (GraphPad Software, La Jolla, CA, USA).

\section{Results}

BDNF exerts biological effects following intracerebroventricular delivery

To assess the effects of BDNF signaling on neuronal protection following infection with $\mathrm{PM}$, the rats were pretreated with exogenous BDNF or the TrkB inhibitor k252a once daily for four consecutive days prior to $S$. pneumoniae exposure. At $24 \mathrm{~h}$ post-infection, the rats were sacrificed (Fig. 1a). Previous studies reported that BDNF exerted an effect on CNS neurons associated with regulating food intake [25]; therefore, we used body weight as an indicator to determine the effects of exogenous BDNF delivery by intracerebroventricular injection. The rats were weighed from the first day of pretreatment, and we observed that their body weight following pretreatment with BDNF increased slowly.

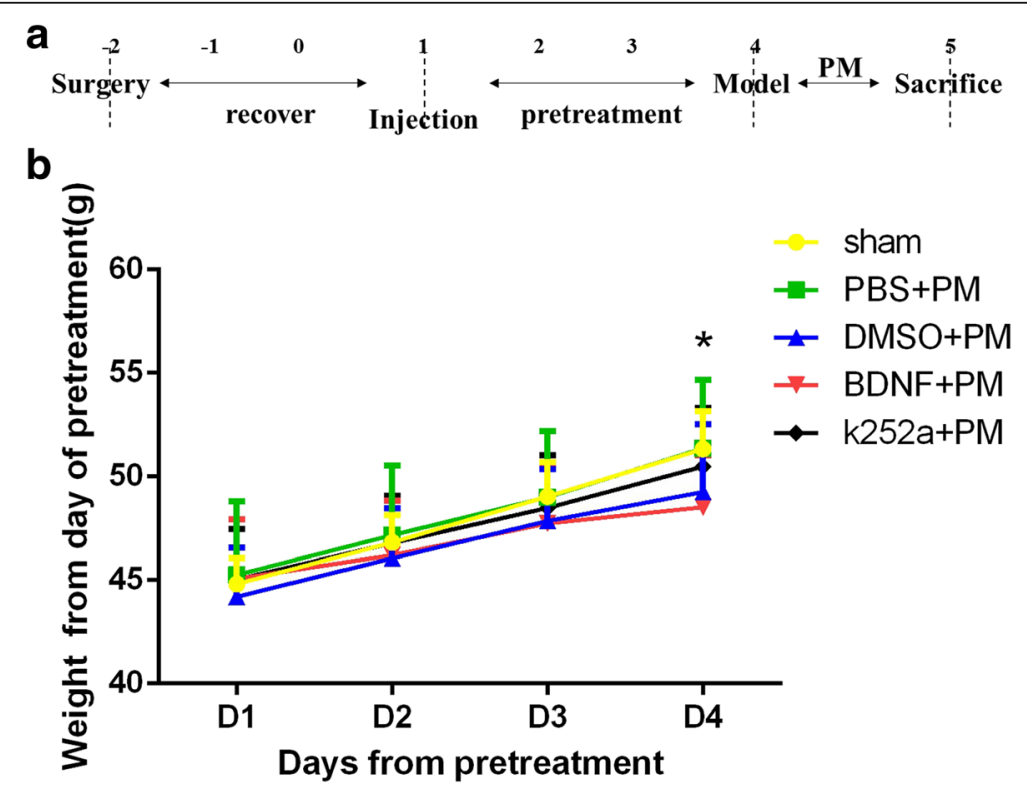

Fig. 1 Exogenous BDNF exerts biological effects following intracerebroventricular delivery. a Experimental procedures. b Rats were weighed from the first day of pretreatment. The body weight of BDNF-pretreated rats increased slowly through day 4, at which time their weight was significantly lower as compared with that of rats in the PBS + PM group. ${ }^{*} p<0.05$, BDNF + PM group compared with the PBS + PM group 
Moreover, on the final day of pretreatment, their body weight was significantly lower than that of rats in the PBS-treated group (Fig. 1b). These results indicated that exogenous BDNF exerted a biological effect following intracerebroventricular delivery.

\section{BDNF upregulates TrkB expression}

We used real-time PCR to evaluate TrkB mRNA levels in the hippocampus (Fig. 2a) and cortex (Fig. 2b) at $24 \mathrm{~h}$ post-inoculation. Our findings showed that TrkB expression increased following infection and that administration of exogenous BDNF further elevated TrkB expression, which agreed with our previous findings related to TrkB response to exogenous BDNF in a PM model [22]. Pretreatment with the TrkB inhibitor k252a attenuated upregulated TrkB expression as compared with that observed in the BDNF + PM group. Western blot analysis of total TrkB protein levels in both the hippocampus (Fig. 2c, e) and cortex (Fig. 2d, f) showed similar trends as those observed in real-time PCR results. These data confirmed results reported in our previous study showing that exogenous BDNF exerted a neuroprotective role by interacting with its receptor, TrkB, following S. pneumoniae meningitis infection [15].

\section{BDNF reduces the clinical severity of PM}

As shown in Table 1, all infected animals yielded positive bacterial cultures from CSF samples, whereas no pneumococci grew from CSF samples obtained from the sham group. Moreover, animals pretreated with BDNF showed lower bacterial titers as compared with those from the PBS + PM group, and all infected animals lost weight $24 \mathrm{~h}$ after infection as compared with the sham group. Additionally, animals pretreated with BDNF exhibited less weight change relative to that observed in the PBS + PM group, whereas animals pretreated with k252a lost more weight as compared with the BDNF + PM group (Table 1). Clinical score evaluation represents
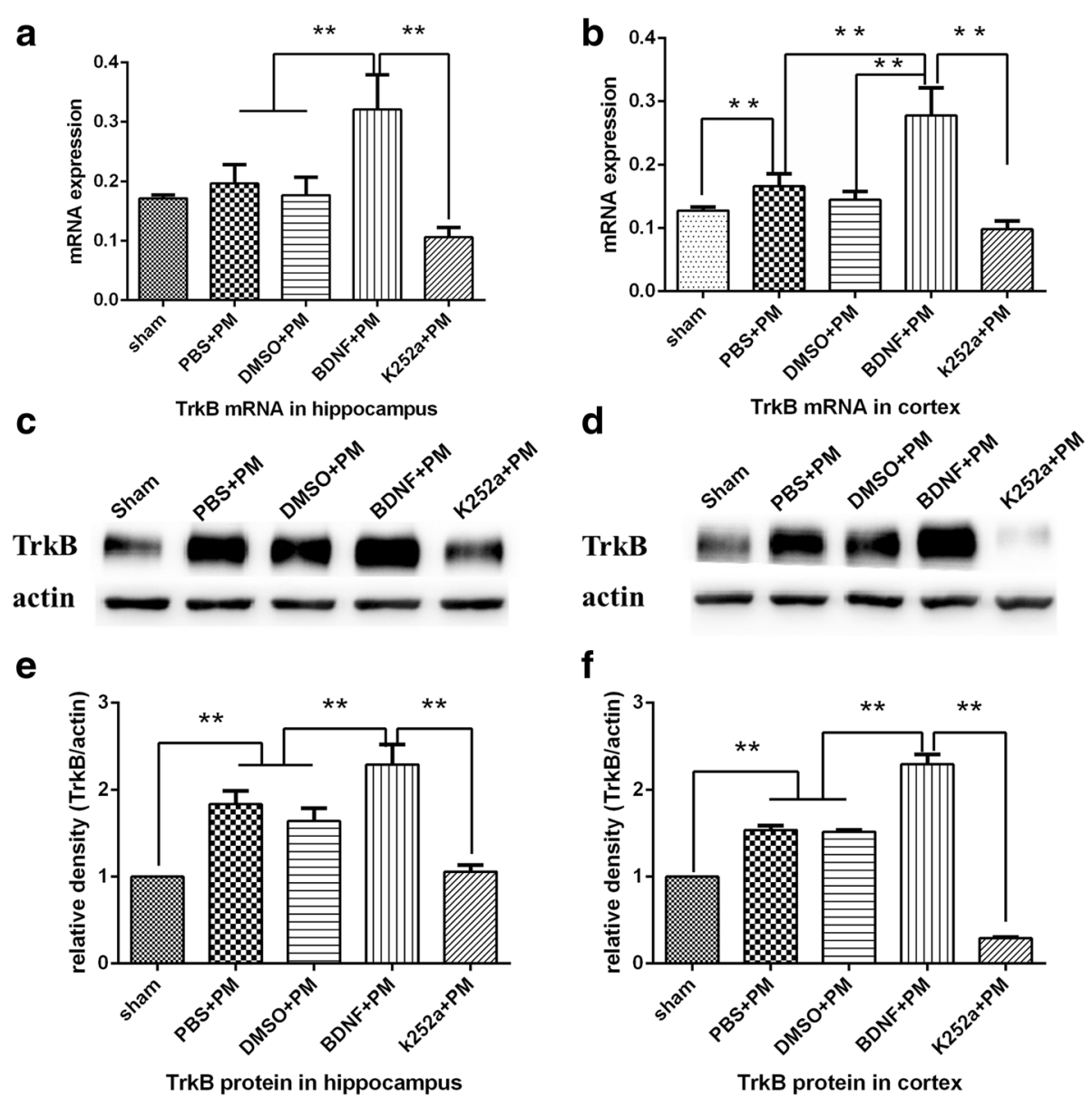

Fig. 2 Exogenous BDNF administration upregulated TrkB expression in both the hippocampus and cortex. Real-time PCR analysis of TrkB mRNA levels in the $\mathbf{a}$ hippocampus and $\mathbf{b}$ cortex at $24 \mathrm{~h}$ post-infection. TrkB mRNA levels significantly increased following infection, with clearly elevated levels observed in the BDNF + PM group. Pretreatment with k252a significantly inhibited TrkB expression relative to levels observed in the BDNF + PM group. Western blot analysis assessed TrkB protein levels in the $\mathbf{c}$, e hippocampus and $\mathbf{d}$, $\mathbf{f}$ cortex showed significantly increased TrkB protein levels following infection. Administration of exogenous BDNF further increased TrkB expression, which was attenuated by treatment with k252a. ${ }^{* *} p<0.01$ 
Table 1 Bacterial titer, change of weight, neurological scores, and mortality in different groups (mean \pm standard deviation)

\begin{tabular}{lllll}
\hline Groups & Bacterial titer, $24 \mathrm{hpi}^{\left[\log _{10} \mathrm{CFU} \mathrm{mL} \mathrm{m}^{-1}\right]}$ & Change of weight & Neurological scores & Mortality $^{\mathrm{a}}$ \\
\hline Sham & - & $-2.64 \pm 0.46$ & $5[5,5]$ & $0 / 10$ \\
PBS + PM & $7.97 \pm 0.50$ & $1.50 \pm 0.39^{*}$ & $3[3,4]^{*}$ & $4 / 14$ \\
DMSO + PM & $7.87 \pm 0.42$ & $1.37 \pm 0.45^{*}$ & $3[3,4]^{*}$ & $3 / 14$ \\
BDNF + PM & $7.35 \pm 0.41^{* *}$ & $1.08 \pm 0.36^{* * * *}$ & $4[3,4]^{*, * *}$ & $2 / 14$ \\
k252a + PM & $8.08 \pm 0.27^{* * *}$ & $2.28 \pm 1.40^{* * * * *}$ & $3[3,4]^{* * * *}$ & $5 / 14$ \\
\hline
\end{tabular}

Bacterial titer, change of weight, neurological scores, and mortality were evaluated at $24 \mathrm{~h}$ post-infection

${ }^{*} p<0.05$, compared to sham group

${ }^{* *} p<0.05$, compared to PBS + PM group

${ }^{* * *} p<0.05$, compared to BDNF + PM group

${ }^{\mathrm{a}}$ Median [minimum, maximum]

an indicator of the degree of disease severity. Table 1 shows that clinical scores were significantly decreased in all PM groups at $24 \mathrm{~h}$ post-inoculation as compared with those measured for the sham group. Additionally, BDNF pretreatment significantly increased the clinical scores of infected rats as compared with those measured in PBS + PM rats. In contrast, rats pretreated with k252a exhibited lower clinical scores as compared with those measured in BDNF + PM rats. These results indicated that BDNF administration attenuated the clinical severity associated with PM. Of the 14 infected animals in each PM group, four (4/14) and three (3/14) animals died in the PBS + PM group and DMSO + PM group, respectively. The survival rate of rats in the BDNF + PM group tended to be higher than that of rats in the PBS + PM group; however, there was no significant difference ( $p>0.05$, log-rank test) (Fig. 3).

\section{BDNF alleviates the pathologic severity of PM}

We investigated morphological changes according to $\mathrm{H} \& \mathrm{E}$ staining at $24 \mathrm{~h}$ post-infection. As shown in Fig. 4, large amounts of inflammatory exudate and infiltrated cells accumulated in the subarachnoid space of all infected groups. Additionally, a significant decrease in the number of cells infiltrated with inflammatory factors was observed in the BDNF + PM group as compared with that observed in the PBS + PM group, although there was no obvious difference between the k252a + PM

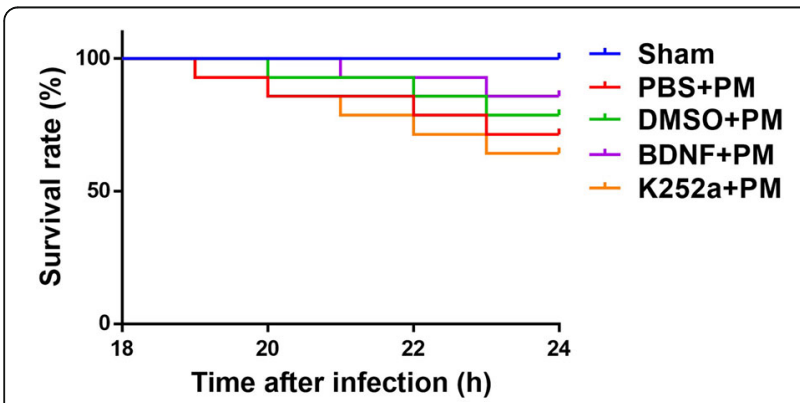

Fig. 3 Kaplan-Meier curves showing the survival rates of animals from different groups group and the DMSO + PM group. These results illustrated the protective effect of BDNF signaling on the pathologic severity of PM.

BDNF administration downregulates proinflammatory cytokine expression and upregulates anti-inflammatory cytokine expression associated with PM infection

Brain homogenates were examined for cytokine (TNF- $\alpha$, IL-1 $\beta$, IL-6, and IL-10) levels by ELISA. The levels of all inflammatory cytokines analyzed were significantly elevated in all the infected rats as compared with those in the sham group. However, the expression of proinflammatory cytokines (TNF- $\alpha$, IL-1 $\beta$, and IL-6) in the hippocampus (Fig. 5a, c, and e) and the cortex (Fig. 5b, d, f) was significantly reduced in the BDNF + PM group as compared with that observed in the PBS + PM group, whereas rats pretreated with $\mathrm{k} 252 \mathrm{a}$ showed elevated inflammatory factor expression relative to that observed in the BDNF + PM group (except for IL-6 in the hippocampus; Fig 5e). In contrast, expression of the antiinflammatory cytokine IL-10 was significantly elevated in both the hippocampus (Fig. 5g) and cortex (Fig. 5h) in the BDNF + PM group as compared with that observed in the PBS + PM group, whereas k252a pretreatment exerted the opposite effect as that observed following BDNF pretreatment. These findings indicated that BDNF/TrkB signaling was significantly associated with the expression of cytokines/chemokines in response to $\mathrm{PM}$ infection.

\section{BDNF inhibits the MyD88/NF-KB-signaling pathway associated with PM}

The MyD88/NF-kB-signaling pathway plays an important role in S. pneumoniae meningitis infection. MyD88 is a critical molecule associated with initiation of host immune response associated with PM [26]. Here, we used real-time PCR to evaluate MyD88 mRNA levels in the hippocampus and cortex at $24 \mathrm{~h}$ post-inoculation and found significant increases in MyD88 mRNA levels in both the hippocampus (Fig. 6a) and cortex (Fig. 6b) of all infected rats. Interestingly, BDNF pretreatment significantly prevented PM- 


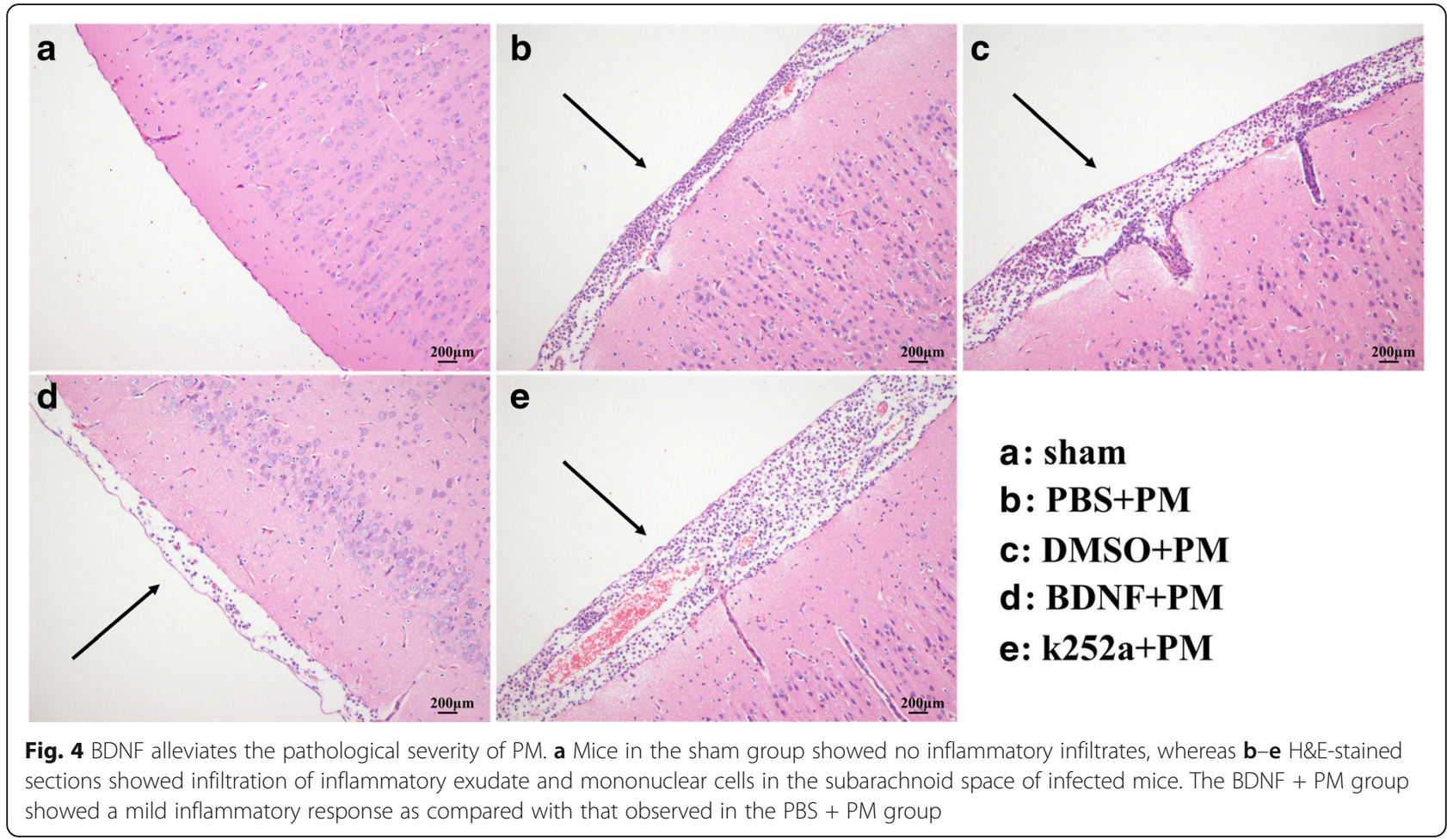

related increases in MyD88 mRNA levels in brain tissues, whereas pretreatment with $\mathrm{k} 252 \mathrm{a}$ significantly increased MyD88 expression as compared with levels observed in the BDNF + PM group. Western blot analysis to evaluate total MyD88 protein levels in the hippocampus (Fig. 6c, e) and cortex (Fig. 6d, f) agreed with mRNA levels measured by real-time PCR.

$\mathrm{NF}-\mathrm{KB}$ is an important transcription factor that plays a key role in the pathophysiological mechanism associated with PM. We performed an EMSA to determine whether exogenous BDNF effects NF-kB-related DNA-binding activity. As shown in Fig. 7a, NF-kB-binding activity in the cortex increased at $24 \mathrm{~h}$ post-inoculation in all $\mathrm{PM}$ groups as compared with that in the sham group. Interestingly, BDNF pretreatment significantly prevented $\mathrm{PM}$-associated elevations in NF-kB-related DNAbinding activity as compared with the PBS + PM group, and pretreatment with k252a significantly increased NF$\kappa \mathrm{B}$-binding activity as compared with activity observed in the BDNF + PM group, although there was no statistical difference between the binding activity observed between the DMSO + PM group and the k252a + PM group (Fig. 7b). These results indicated that BDNF/TrkB signaling inhibited activity associated with the MyD88/ NF-кB-signaling pathway associated with PM.

\section{BDNF reduces hippocampal apoptosis in PM}

Hippocampal apoptosis was investigated by TUNEL staining. Our results indicated that all PM groups exhibited increased levels of apoptosis as compared with those in the sham group, with most apoptotic cells mainly located in the inner layer of the dentate (Fig. 8a). Furthermore, rats that received BDNF pretreatment showed lower levels of apoptosis in the hippocampal dentate gyrus as compared with the PBS + PM group, and rats pretreated with k252a showed an obviously increased number of apoptotic cells as compared with that observed in the BDNF + PM group (Fig. 8b).

\section{BDNF activates the PI3K/AKT-signaling pathway in PM}

To examine whether exogenous BDNF activates PI3K/ AKT signaling in $\mathrm{PM}$, we measured the levels of phosphorylated PI3K (p-PI3K) and AKT (p-AKT). Figure 9 shows that levels of p-PI3K and p-AKT were significantly increased in infected brain tissues as compared with those observed in the sham control, although the total protein levels of PI3K and AKT were unchanged. Additionally, administration of exogenous BDNF significantly increased p-PI3K and p-AKT levels in both the hippocampus (Fig. 9a, b, e, f) and cortex (Fig. 9c, d, g, h) as compared with the levels observed in the PBS + PM group. However, rats pretreated with k252a exhibited significantly lower levels of PI3K and AKT phosphorylation relative to the levels observed in the BDNF + PM group. These data indicated that BDNF/TrkB signaling activated the PI3K/AKT-signaling pathway in PM via PI3K and AKT phosphorylation. 

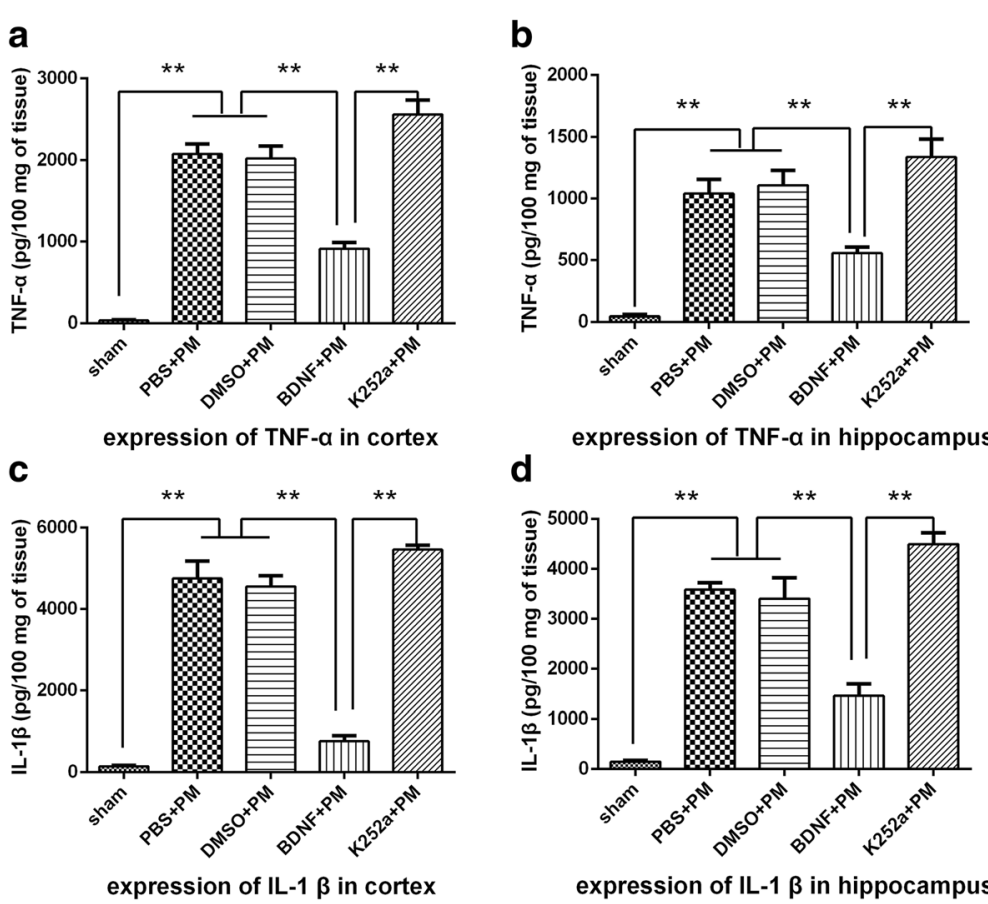

d

\section{expression of TNF- $\alpha$ in hippocampus}

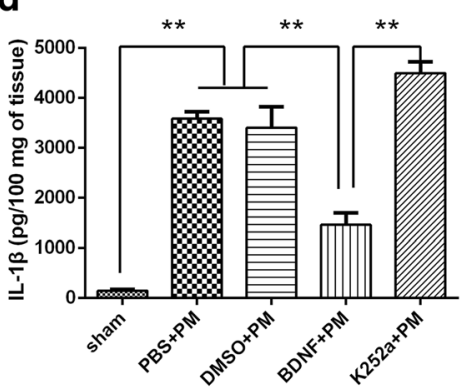

expression of IL-1 $\beta$ in hippocampus

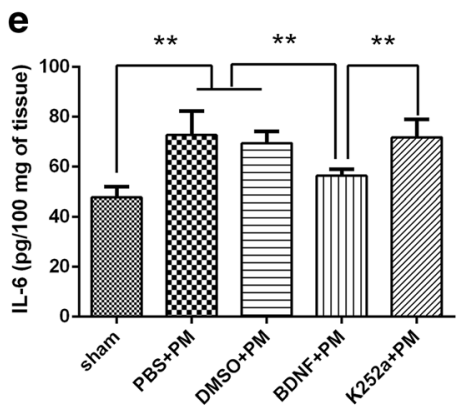

f
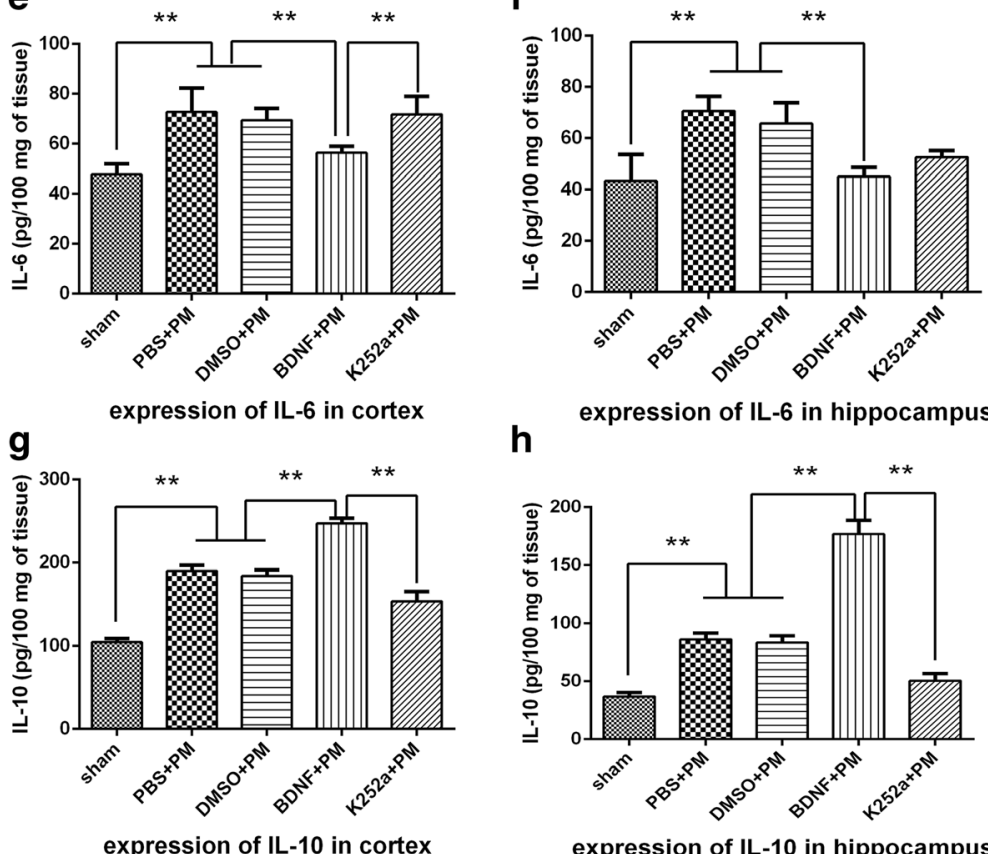

h

expression of IL- 6 in hippocampus

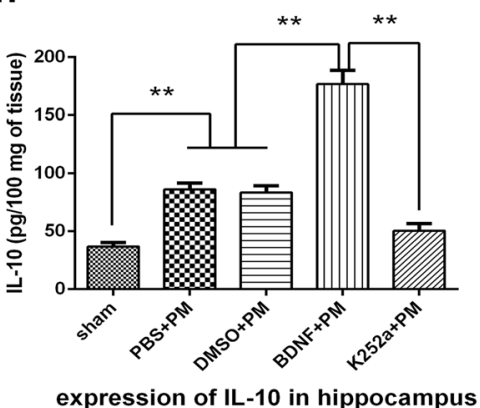

Fig. 5 BDNF downregulates proinflammatory cytokine expression and upregulates anti-inflammatory cytokine expression in PM. Levels of proinflammatory cytokines (TNF-a, IL-1 $\beta$, and IL-6) and anti-inflammatory cytokines (IL-10) in both the hippocampus and cortex at $24 \mathrm{~h}$ post-infection as measured by ELISA. Levels of both proinflammatory and anti-inflammatory cytokines were significantly increased at $24 \mathrm{~h}$ post-infection as compared with levels observed in the sham group. Administration of exogenous BDNF significantly decreased the levels of TNF- $a, I L-1 \beta$, and IL- 6 in the $\mathbf{a}, \mathbf{c}$, e hippocampus and $\mathbf{b}, \mathbf{d}, \mathbf{f}$ cortex, and $\mathbf{g}, \mathbf{h}$ further elevated IL-10 levels. Rats pretreated with k252a exhibited enhanced expression of proinflammatory cytokines and attenuated expression of anti-inflammatory cytokines as compared with levels observed in the BDNF + PM group. ${ }^{* *} p<0.01$

\section{Discussion}

In this study, we observed that BDNF pretreatment reduced the clinical and pathological severity of PM and also alleviated the inflammatory response and hippocampal apoptosis associated with S. pneumoniae meningitis. Additionally, we demonstrated that downregulated BDNF/ TrkB interactions following treatment with a TrkBspecific pharmacological inhibitor reversed the effects 

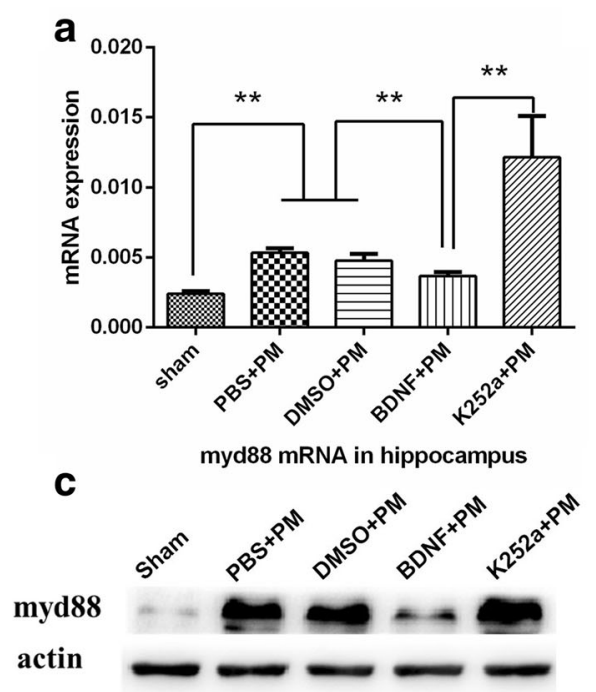

e

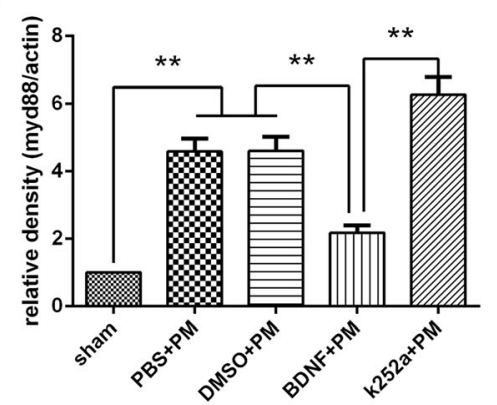

myd88 protein in hippocampus

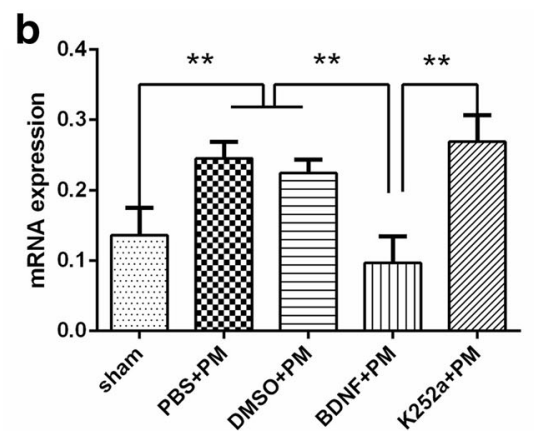

d

$$
\text { myd88 mRNA in cortex }
$$

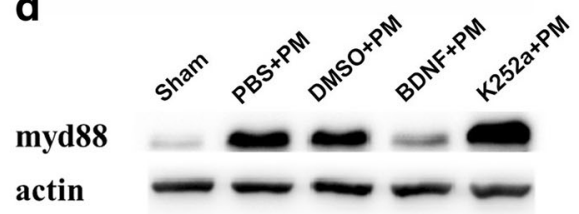

f

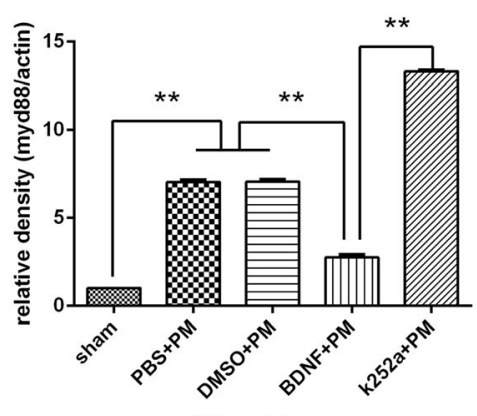

myd88 protein in cortex

Fig. 6 BDNF inhibited MyD88 expression in PM. MyD88 mRNA levels in the a hippocampus and $\mathbf{b}$ cortex increased at $24 \mathrm{~h}$ post-infection. BDNF pretreatment inhibited the upregulation of MyD88 mRNA, whereas rats pretreated with k252a showed increased MyD88 expression as compared with that observed in the BDNF + PM group. Western blot analysis confirmed lower levels of MyD88 protein in the $\mathbf{c}$, e hippocampus and $\mathbf{d}, \mathbf{f}$ cortex following BDNF treatment, whereas these results were not observed in the k252a + PM group. ${ }^{* *} p<0.01$
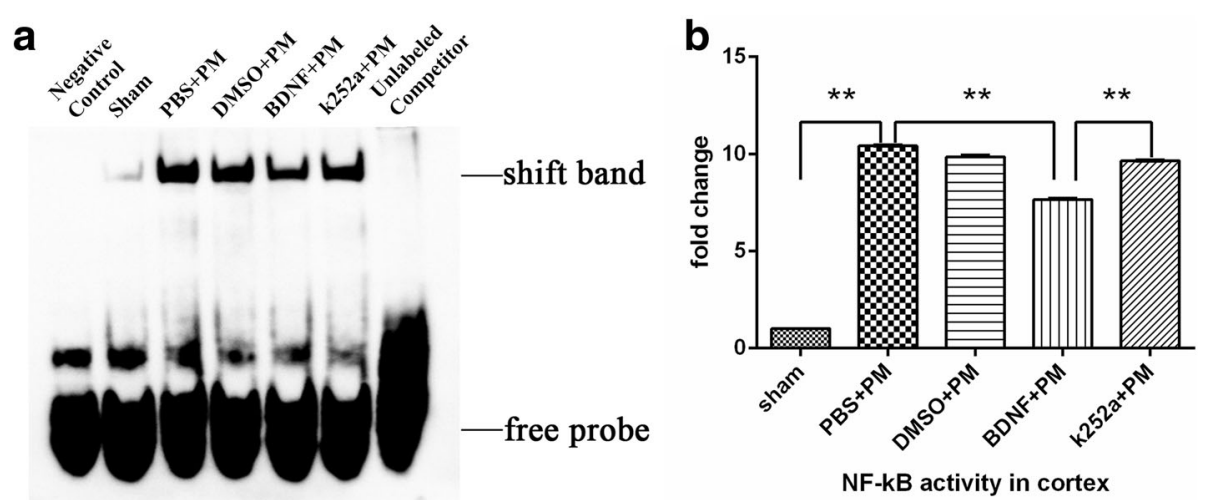

Fig. 7 BDNF reduces NF-KB-binding activity in the cortex at $24 \mathrm{~h}$ post-infection. a NF-KB DNA-binding activity examined by EMSA revealed increased NFKB-binding activity in all infected mice relative to levels observed in the sham group. BDNF pretreatment inhibited NF-KB-binding activity as compared with levels observed in the PBS + PM group, and pretreatment with k252a significantly increased NF-kB-binding activity as compared with activity observed in the BDNF + PM group. $\mathbf{b}$ Quantitative analysis of the EMSA results. ${ }^{*} p<0.01$ 


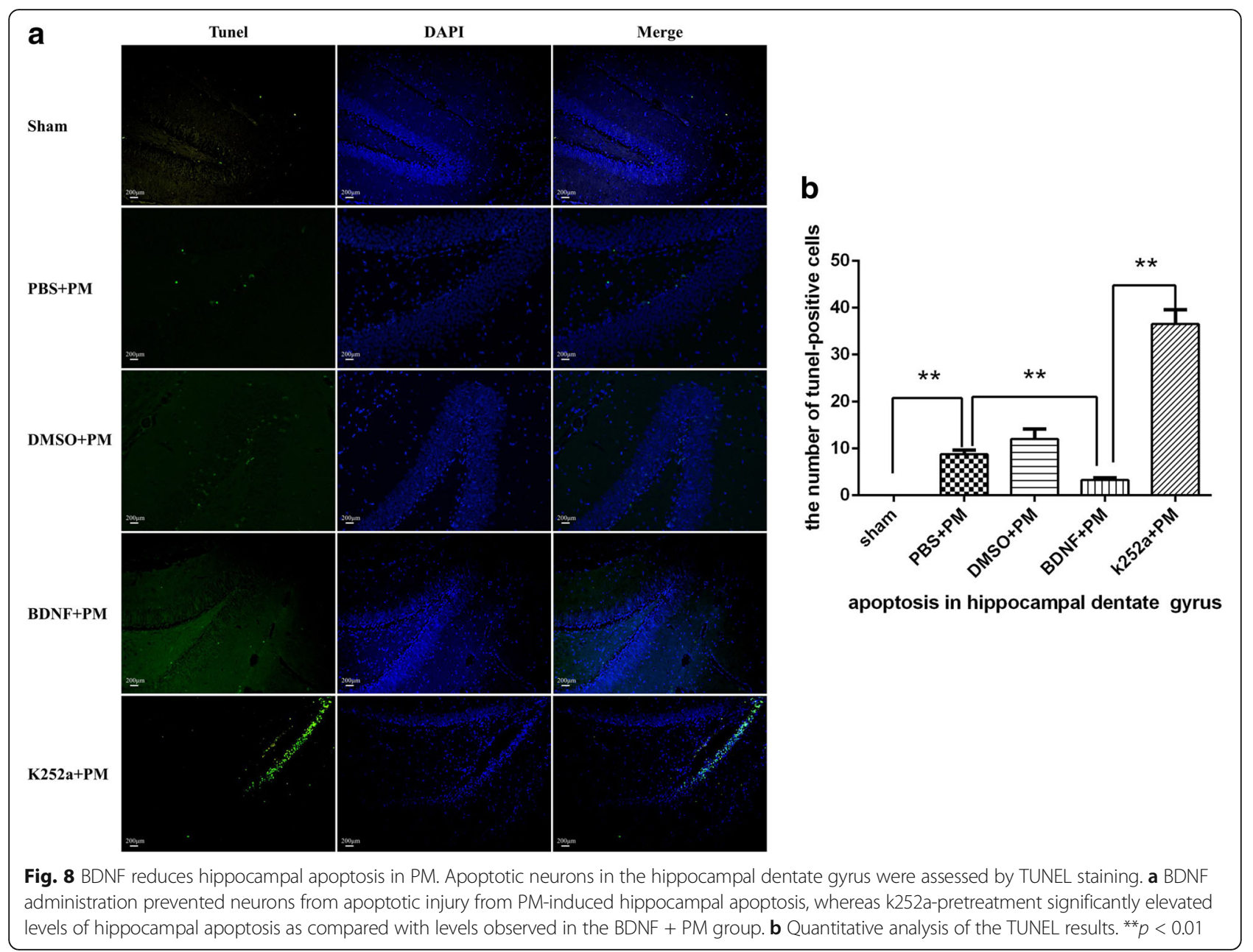

observed following BDNF treatment. Furthermore, based on investigation of intracellular signaling pathways, our results indicated that the neuroprotective effects exerted by BDNF might occur through modulation of the MyD88/ NF-KB- and PI3K/AKT-signaling pathways.
Despite the advances in antimicrobial agents and improved neurological management, it remains difficult to improve outcomes for children suffering from bacterial meningitis [27]. The discovery of the neuroprotective effects associated with BDNF in diverse CNS-related

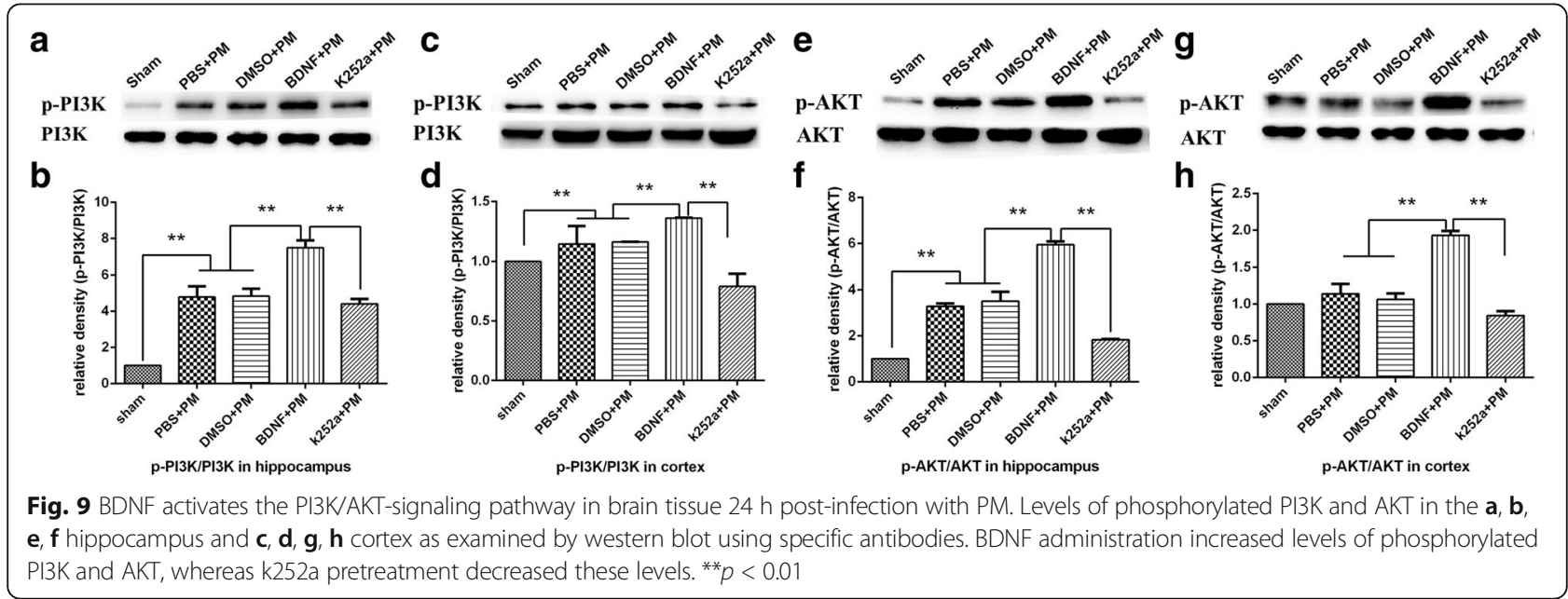


diseases offers a potentially promising strategy for treating paradigms of acute neuroinfection. Although BDNF expression is increased transiently in the brains during the acute phase of meningitis [15], this endogenous protective mechanism is insufficient to protect neurons from infection-related death. Early studies reported that adjuvant therapy involving BDNF protects the brain from three distinct forms of injury in experimental bacterial meningitis [28]; however, here, we investigated the effects of BDNF/TrkB interaction on the inflammatory response and hippocampal apoptosis, as well as its effect on other potential intracellular signaling pathways responsible for this protective activity associated with PM.

During the acute phase of the disease, inflammatory mediators are produced and released by brain-resident immune cells and different neuron populations, thereby contributing to disease pathogenesis $[29,30]$. Proinflammatory cytokines, such as TNF- $\alpha$, IL- $1 \beta$, and interferon$\gamma$, play a key role in disease progression during diverse types of CNS insult, eventually resulting in depleted energy production, cell death, and brain injury [31-33]. In this study, we observed that that BDNF treatment decreased both mRNA and protein levels of TNF- $\alpha$, IL- $1 \beta$, and IL- 6 induced by the presence and recognition of bacterial components. These results might partially explain the reduced pathological severity observed in our $\mathrm{H} \& \mathrm{E}$ staining results. IL-10 is an anti-inflammatory cytokine that exhibits neuroprotective effects based on studies reporting that exogenous administration of IL-10 protects the brains from ischemic injury [34] and increased severity of brain injury in IL-10 knockout mice suffering from multiple sclerosis [35]. Here, exogenous BDNF administration increased IL-10 expression at the transcription and translation levels, suppressed proinflammatory cytokine expression, and enhanced antiinflammatory cytokine expression in bacterial meningitis models, which was consistent with observations from previous studies $[19,36]$. A recent in vivo study showed that BDNF was capable of regulating intracellular signaling molecules to inhibit inflammatory cytokine expression induced by peptidoglycan in human dental pulp cells [37]. Despite these findings, the exact mechanism by which BDNF regulates anti-inflammatory effects during bacterial meningitis infection remains unclear.

The MyD88/NF- $\mathrm{kB}$ complex forms a classic inflammatory signaling pathway critical to responses to CNS insult. BDNF prevents neurological deficiency in focal cerebral ischemia by suppressing the toll-like receptor/ MyD88-signaling pathway [14], and in PM, the MyD88/ NF- $\mathrm{kB}$-signaling pathway is activated by recognition of bacterial compounds [38]. Here, we observed that BDNF pre-treatment inhibited the expression of MyD88 and nuclear translocation of NF-kB, thereby neutralizing the detrimental effects of excessive inflammatory responses in suggesting that BDNF administration might enable preservation of neurons from PM-related injury.

Previous studies revealed three major forms of brain injury according to PM-related histopathology results: necrosis of the cortex, apoptosis in the hippocampus, and neuronal loss in the spiral ganglion [28]. The hippocampal dentate gyrus is particularly vulnerable to apoptotic injury during PM and usually appears $\sim 18$ to $\sim 24 \mathrm{~h}$ post-infection and is accompanied by massive increases in the number of apoptotic cells [39], with most apoptotic cells located in the inner layer of the dentate gyrus, which is consistent with our results [40]. The neuroprotective effects of BDNF were described in a study focused on neonatal hypoxia-ischemia, wherein BDNF prevented hippocampal apoptosis by blocking caspase-3 activation [41]. BDNF/TrkB interaction induces complex intracellular signal-transduction cascades, including the mitogen-activated protein kinase pathway, PI3K/AKT pathway, and phospholipase C-gamma pathway [42]. Additionally, previous studies reported that BDNF delivered by bone marrow stem cells inhibited apoptosis in both multiple sclerosis and ischemic stroke models through upregulated expression of anti-apoptotic B cell lymphoma 2 (BCL-2) and downregulated expression of pro-apoptotic BCL-2-associated $\mathrm{X}$ protein (BAX). Because PI3K/AKT signaling occurs upstream of BCL-2/ $\mathrm{BAX}$ expression/repression, we hypothesized that the anti-apoptotic effects associated with BDNF/TrkB interactions might involve the PI3K/AKT pathway. The results of our study showed that PI3K/AKT signaling was significantly upregulated after meningitis infection and that this activity was enhanced following BDNF pretreatment. As an additional pathway associated with cell survival, excess concentrations of cellular calcium represent a common pathophysiological phenomenon during bacterial meningitis [43]. Activity associated with PI3K/AKT signaling is capable of regulating calcium channels to enhance their capacity to bind intracellular calcium, thereby promoting cellular calcium homeostasis and survival [44].

\section{Conclusions}

In summary, our findings indicated that BDNF/TrkB interactions resulted in anti-inflammatory and antiapoptotic effects in experimental models of PM likely through regulation of the MyD88/NF-kB- and PI3K/AKTsignaling pathways. These results suggested that BDNF might constitute a promising therapeutic alternative for the attenuation of PM-associated neurofunctional deficits.

\footnotetext{
Abbreviations

AKT: Protein kinase B; BDNF: Brain-derived neurotrophic factor; CNS: Central nervous system; CSF: Cerebrospinal fluid; DMSO: Dimethyl sulfoxide; ELISA: Enzyme-linked immunosorbent assay; EMSA: Electrophoretic mobility shift assay; ERK: Extracellular signal-related kinase; H\&E: Hematoxylin and eosin; IL: Interleukin; MyD88: Myeloid differentiation primary response gene 88; NFKB: Nuclear factor kappa B; PBS: Phosphate-buffered saline; PCR: Polymerase
} 
chain reaction; PFA: Paraformaldehyde; PI3K: Phosphatidylinositol 3-kinase; PM: Pneumococcal meningitis; S. pneumoniae: Streptococcus pneumoniae; TBST: Tris-buffered saline and Tween-20; TNF-a: Tumor necrosis factor-a; TrkB: Tropomyosin-receptor kinase B; TUNEL: tissue pathology and terminal deoxynucleotidyl transferase dUTP-nick-end labeling

\section{Acknowledgements}

Not applicable.

\section{Funding}

This work was supported by the National Natural Science Foundation of China (grant numbers 81571180 and 81271337 ).

\section{Availability of data and materials}

The datasets and materials supporting the conclusions of this article are included within the article.

\section{Authors' contributions}

DX wrote the main manuscript text. DL performed the in vivo experiment of the study. JW helped with the animal experiment. YL cultured the infectious organisms for the study. JS and MZ performed the histopathological experiments of the study. DH performed the molecular biological experiments. LL provided the necessary guidance on the performance of all the experiment. All authors read and approved the final article.

\section{Ethics approval and consent to participate}

The animal experiments were approved by the Animal Ethical and Welfare Committee of Xinhua Hospital (approval ID: 2014041).

\section{Consent for publication}

Not applicable.

\section{Competing interests}

The authors declare that they have no competing interests.

\section{Publisher's Note}

Springer Nature remains neutral with regard to jurisdictional claims in published maps and institutional affiliations.

\section{Author details}

${ }^{1}$ Department of Pediatric Neurology, Xinhua Hospital affiliated to Shanghai Jiaotong University School of Medicine, Kongjiang Rd 1665, Shanghai 200092, People's Republic of China. ²Department of Clinical Laboratory, Xinhua Hospital affiliated to Shanghai Jiaotong University School of Medicine, Shanghai 200092, People's Republic of China. ${ }^{3}$ Department of Pathology, Xinhua Hospital affiliated to Shanghai Jiaotong University School of Medicine, Shanghai 200092, People's Republic of China.

Received: 5 March 2017 Accepted: 27 July 2017

Published online: 04 August 2017

\section{References}

1. Ceyhan M, Ozsurekci Y, Gurler N, et al. Bacterial agents causing meningitis during 2013-2014 in Turkey: a multi-center hospital-based prospective surveillance study. Hum Vaccin Immunother. 2016:12:2940-5.

2. Brouwer MC, Tunkel AR, van de Beek D. Epidemiology, diagnosis, and antimicrobial treatment of acute bacterial meningitis. Clin Microbiol Rev. 2010;23:467-92.

3. van de Beek D, de Gans J, Spanjaard L, et al. Clinical features and prognostic factors in adults with bacterial meningitis. N Engl J Med. 2004;351:1849-59.

4. van de Beek D, Farrar JJ, de Gans J, et al. Adjunctive dexamethasone in bacterial meningitis: a meta-analysis of individual patient data. Lancet Neurol. 2010:9:254-63.

5. van de Beek D, Brouwer M, Hasbun R, et al. Community-acquired bacterial meningitis. Nat Rev Dis Primers. 2016;2:16074.

6. Lucas MJ, Brouwer MC, van de Beek D. Neurological sequelae of bacterial meningitis. J Inf Secur. 2016;73:18-27.

7. Sellner J, Tauber MG, Leib SL. Pathogenesis and pathophysiology of bacterial CNS infections. Handb Clin Neurol. 2010;96:1-16.

8. Quesada SJ. Social medicine and socialization of medicine. An R Acad Nac Med (Madr). 1970;87:169-229.
9. Lian D, He D, Wu J, et al. Exogenous BDNF increases neurogenesis in the hippocampus in experimental Streptococcus pneumoniae meningitis. J Neuroimmunol. 2016;294:46-55.

10. Koedel U, Scheld WM, Pfister HW. Pathogenesis and pathophysiology of pneumococcal meningitis. Lancet Infect Dis. 2002;2:721-36.

11. Horch HW, Kruttgen A, Portbury SD, et al. Destabilization of cortical dendrites and spines by BDNF. Neuron. 1999;23:353-64.

12. Huang EJ, Reichardt LF. Neurotrophins: roles in neuronal development and function. Annu Rev Neurosci. 2001;24:677-736.

13. Makar TK, Trisler D, Sura KT, et al. Brain derived neurotrophic factor treatment reduces inflammation and apoptosis in experimental allergic encephalomyelitis. J Neurol Sci. 2008;270:70-6.

14. Lu H, Liu X, Zhang N, et al. Neuroprotective effects of brain-derived neurotrophic factor and noggin-modified bone mesenchymal stem cells in focal cerebral ischemia in rats. J Stroke Cerebrovasc Dis. 2016:25:410-8.

15. Li L, Shui QX, Zhao ZY. Regulation of brain-derived neurotrophic factor (BDNF) expression following antibiotic treatment of experimental bacterial meningitis. J Child Neurol. 2003;18:828-34.

16. Morichi $S$, Kashiwagi $Y$, Takekuma $K$, et al. Expressions of brain-derived neurotrophic factor (BDNF) in cerebrospinal fluid and plasma of children with meningitis and encephalitis/encephalopathy. Int J Neurosci. 2013;123: 17-23.

17. Tauber SC, Stadelmann C, Spreer A, et al. Increased expression of BDNF and proliferation of dentate granule cells after bacterial meningitis. J Neuropathol Exp Neurol. 2005;64:806-15.

18. Li L, Shui QX, Liang K, et al. Brain-derived neurotrophic factor rescues neurons from bacterial meningitis. Pediatr Neurol. 2007;36:324-9.

19. Jiang $Y$, Wei N, Lu T, et al. Intranasal brain-derived neurotrophic factor protects brain from ischemic insult via modulating local inflammation in rats. Neuroscience. 2011:172:398-405.

20. Crowder RJ, Freeman RS. Phosphatidylinositol 3-kinase and Akt protein kinase are necessary and sufficient for the survival of nerve growth factordependent sympathetic neurons. J Neurosci. 1998;18:2933-43.

21. Han $\mathrm{BH}$, Holtzman DM. BDNF protects the neonatal brain from hypoxicischemic injury in vivo via the ERK pathway. J Neurosci. 2000;20:5775-81.

22. Song $X$, Lian $D$, He $D$, et al. The responsiveness of TrkB to exogenous BDNF in frontal cortex during antibiotic treatment of Streptococcus pneumoniae meningitis. Neurol Sci. 2014;35:1915-23.

23. Leib SL, Clements JM, Lindberg RL, et al. Inhibition of matrix metalloproteinases and tumour necrosis factor alpha converting enzyme as adjuvant therapy in pneumococcal meningitis. Brain. 2001;124:1734-42.

24. Wei HJ, Xu JH, Li MH, et al. Hydrogen sulfide inhibits homocysteine-induced endoplasmic reticulum stress and neuronal apoptosis in rat hippocampus via upregulation of the BDNF-TrkB pathway. Acta Pharmacol Sin. 2014;35: 707-15.

25. Lapchak PA, Hefti F. BDNF and NGF treatment in lesioned rats: effects on cholinergic function and weight gain. Neuroreport. 1992:3:405-8.

26. Koedel U, Rupprecht T, Angele B, et al. MyD88 is required for mounting a robust host immune response to Streptococcus pneumoniae in the CNS. Brain. 2004;127:1437-45

27. Gounder PP, Zulz T, Desai $\mathrm{S}$, et al. Epidemiology of bacterial meningitis in the North American Arctic, 2000-2010. J Inf Secur. 2015;71:179-87.

28. Bifrare YD, Kummer J, Joss $P$, et al. Brain-derived neurotrophic factor protects against multiple forms of brain injury in bacterial meningitis. J Infect Dis. 2005; 191:40-5.

29. van de Beek D. Corticosteroids for acute adult bacterial meningitis. Med Mal Infect. 2009:39:531-8.

30. Barichello T, dos Santos I, Savi GD, et al. TNF-alpha, IL-1 beta, IL-6, and cinc-1 levels in rat brain after meningitis induced by Streptococcus pneumoniae. Neuroimmunol. 2010:221:42-5.

31. Hofer $S$, Grandgirard D, Burri D, et al. Bacterial meningitis impairs hippocampal neurogenesis. J Neuropathol Exp Neurol. 2011:70:890-9.

32. Codarri L, Fontana A, Becher B. Cytokine networks in multiple sclerosis: lost in translation. Curr Opin Neurol. 2010:23:205-11.

33. Gerber J, Nau R. Mechanisms of injury in bacterial meningitis. Curr Opin Neurol. 2010;23:312-8.

34. Ooboshi $H$, Ibayashi S, Shichita T, et al. Postischemic gene transfer of interleukin-10 protects against both focal and global brain ischemia. Circulation. 2005:111:913-9.

35. Bettelli E, Das MP, Howard ED, et al. IL-10 is critical in the regulation of autoimmune encephalomyelitis as demonstrated by studies of IL-10- and IL-4-deficient and transgenic mice. J Immunol. 1998;161:3299-306. 
36. Makar TK, Bever CT, Singh IS, et al. Brain-derived neurotrophic factor gene delivery in an animal model of multiple sclerosis using bone marrow stem cells as a vehicle. J Neuroimmunol. 2009;210:40-51.

37. Takeda K, Tokunaga N, Aida Y, et al. Brain-derived neurotrophic factor inhibits peptidoglycan-induced inflammatory cytokine expression in human dental pulp cells. Inflammation. 2017:40:240-7.

38. Liu S, Kielian T. MyD88 is pivotal for immune recognition of Citrobacter koseri and astrocyte activation during CNS infection. J Neuroinflammation. 2011;8:35.

39. Gianinazzi C, Grandgirard D, Imboden H, et al. Caspase-3 mediates hippocampal apoptosis in pneumococcal meningitis. Acta Neuropathol. 2003;105:499-507.

40. Grandgirard D, Bifrare YD, Pleasure SJ, et al. Pneumococcal meningitis induces apoptosis in recently postmitotic immature neurons in the dentate gyrus of neonatal rats. Dev Neurosci. 2007:29:134-42.

41. Han BH, D'Costa A, Back SA, et al. BDNF blocks caspase-3 activation in neonatal hypoxia-ischemia. Neurobiol Dis. 2000;7:38-53.

42. Chao MV. Neurotrophins and their receptors: a convergence point for many signalling pathways. Nat Rev Neurosci. 2003;4:299-309.

43. Leib SL, Kim YS, Ferriero DM, et al. Neuroprotective effect of excitatory amino acid antagonist kynurenic acid in experimental bacterial meningitis. J Infect Dis. 1996:173:166-71.

44. Alvin Z, Laurence GG, Coleman BR, et al. Regulation of L-type inward calcium channel activity by captopril and angiotensin II via the phosphatidyl inositol 3kinase pathway in cardiomyocytes from volume-overload hypertrophied rat hearts. Can J Physiol Pharmacol. 2011;89:206-15.

\section{Submit your next manuscript to BioMed Central} and we will help you at every step:

- We accept pre-submission inquiries

- Our selector tool helps you to find the most relevant journal

- We provide round the clock customer support

- Convenient online submission

- Thorough peer review

- Inclusion in PubMed and all major indexing services

- Maximum visibility for your research

Submit your manuscript at www.biomedcentral.com/submit 